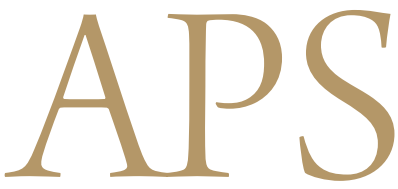

Archives of Plastic Surgery

\title{
The Story of Serratia Marcescens: Pathologic Risk Factors in Breast Implant Surgery
}

\author{
Caroline A Yao ${ }^{1}$, Diana Wang ${ }^{1}$, David A Kulber ${ }^{1,2}$ \\ ${ }^{I}$ Division of Plastic and Reconstructive Surgery, Keck School of Medicine of the University of Southern California, Los Angeles; ${ }^{2}$ Center for \\ Plastic and Reconstructive Surgery, Department of Surgery, Cedars-Sinai Medical Center, Los Angeles, CA, USA
}

Serratia marcescens (S. marcescens) emerged as an opportunist in the setting of immunodeficiency in the 1970s, when serious infections occurred in San Francisco hospitals after USA. Navy experiments had aerosolized the bacteria to study biologic warfare. We investigate the risks of S. marcescens in San Franciscans who undergo mastectomy with implant reconstruction. From 2007 to 2011, the senior author took breast capsule cultures for all patients at the time of tissue expander exchange/explant. Of the 142 women who had reconstruction, 23 had positive cultures. Only the two patients who were positive for S. marcescens developed clinical infections that required explantation. Both had postoperative chemotherapy with transient neutropenia, and both had close ties to San Francisco. Clinical signs of infection emerged for both patients months after initial surgery, despite having previously well healed incisions. Other patients were culture positive for Pseudomonas, Proteus, Enterococcus and MRSA and did not develop require explant. While the link between San Francisco and S. marcescens is controversial, a patient's geography is a simple screening tool when considering postoperative risks, especially in the immunocompromised. Closer monitoring for neutropenia during chemotherapy, and a lower threshold to administer S. marcescens targeted antibiotics may be warranted in these patients.

Keywords Breast implant / Serratia / Device removal / Infection / San francisco

\author{
Correspondence: David A Kulber \\ Cedars Sinai Medical Center, \\ 8635 W. Third Street, Suite 990W, \\ Los Angeles, CA 90048, USA \\ Tel: +1-310-423-5900 \\ Fax: +1-323-442-7901 \\ E-mail: David.Kulber@cshs.org
}

\begin{abstract}
This article was presented at The American College of Surgeons (Southern California Chapter) Annual Scientific Meeting: The Story of Serratia Marcescens: Pathologic Risk Factors in Breast Implant Surgery on January 20, 2012 in Santa Barbara, CA, USA.
\end{abstract}

No potential conflict of interest relevant to this article was reported.

\section{INTRODUCTION}

Before the 1970s, scientists used Serratia marcescens (S. marcescens) as an easily detected biomarker because of its distinctive red pigmentation. It was regularly sprayed on the hands and lips of test subjects, aerosolized in hospital ventilation systems and used as a biomarker in blood [1]. From 1949 to 1968, US Army and Navy experiments in San Francisco aerosolized the bacteria to study the behavior/viability of biologic weapons over large geographic areas; within days, the bacteria was detected 80 meters inland. Shortly after experiments in September 1950 [2,3], local concerns arose when Wheat et al. [4] reported 11 patients who developed S. marcescens urinary tract infections after cystoscopy or prostatectomy. Classified government documents regarding the aerosolization experiments were publically released after the death of one of the patients in Wheat's report who developed bactermia.

S. marcescens affected immunocompromised residents of San Francisco repeatedly in the 1960s and 1970s. Nineteen cases of S. marcescens endocarditis and one case of S. marcescens vertebral osteomyelitis were reported in intravenous drug users [5,6]. Authors of these reports suggested that the government experi- 
ments were a possible explanation for these unusual infections in the community. Though it was not possible to prove a direct link, public concern about the government experiments heightened. Public hearings by the United States Senate Subcommittee on Health and Scientific Research began in 1977 and the use of aerosolized experiments terminated in 1979.

Today, S. marcescens as a virulent agent in nosocomial infections; it thrives in moist environments and spreads through water droplets and contact. Breast cancer patients who undergo chemotherapy and radiation are a unique, immunocompromised group and we investigate the risks of $S$. marcescens in those who received tissue expander-based reconstruction. This paper reviews a series of cases with cultures positive for S. marcescens and identifies risk factors, including geographic, for pathogenic sequelae of $S$. marcescens.

\section{Methods}

Between 2007 and 2011, 142 women underwent mastectomy for breast cancer and had immediate subpectoral tissue expander placement with or without acellular dermal matrix (ADM) by the senior author. After the desired tissue expander size was obtained using weekly saline injections, (one to four months after placement), implant reconstruction was done using silicone prostheses. The senior author routinely takes breast capsule cultures at the time of tissue expander exchange or explant. Patient demographics, cancer treatment and surgical details were recorded. Inclusion criteria for this study were all patients who received immediate tissue expander-based reconstruction for mastectomy, with subsequent implant exchange. Descriptive statistics of patient demographics, culture results and surgical complications were performed using Stata (ver. 11.0, StataCorp LP, College Station, TX, USA).

\section{CASE}

Twenty-three of 142 women had positive breast capsule cultures, including Enterococcus, S. marcescens, Pseudomonas aeruginosa, Methicillin-resistant Staphylococcus aureus (MRSA), Proteus mirabilis and E. coli. 11 of the 23 culture positive patients had ADM with tissue expander placement. Table 1 shows patient clinical details and culture results.

Only the two patients with breast capsules positive for S. marcescens developed clinical infections that required explantation. Both patients with S. marcescens had immediate reconstruction with $\mathrm{ADM}$ and received immediate postoperative chemotherapy that caused transient but significant neutropenia. Additional-

\section{Table 1. Patient clinical details}

\begin{tabular}{|c|c|c|c|c|c|c|c|c|c|c|}
\hline Patient & $\begin{array}{l}\text { Age } \\
\text { (yr) }\end{array}$ & Culture & Complication(s) & Alloderm & $\begin{array}{l}\text { Diabetes } \\
\text { mellitus II }\end{array}$ & $\begin{array}{l}\text { Hyper- } \\
\text { tension }\end{array}$ & Smoker & $\begin{array}{l}\text { Immediate } \\
\text { reconstruction }\end{array}$ & Radiation & $\begin{array}{l}\text { Chemo- } \\
\text { therapy }\end{array}$ \\
\hline 1 & 60 & E. Coli & Small dehiscence & - & $\vee$ & & $\vee$ & - & $\vee$ & - \\
\hline 2 & 62 & Enterococcus & Cellulitis & - & - & $\vee$ & - & $\vee$ & - & - \\
\hline 3 & 41 & Enterococcus & - & - & - & & - & $\vee$ & - & $\vee$ \\
\hline 4 & 57 & MRSA & Cellulitis & $\vee$ & - & - & - & $\vee$ & - & - \\
\hline 5 & 57 & MRSA & Cellulitis & $\vee$ & - & & - & $\vee$ & $\vee$ & - \\
\hline 6 & 73 & Propionibacterium & - & - & - & v & - & $\vee$ & - & - \\
\hline 7 & 40 & Propionibacterium & - & $\vee$ & - & - & - & $\vee$ & - & - \\
\hline 8 & 45 & Propionibacterium & - & $\vee$ & - & - & - & v & - & - \\
\hline 9 & 47 & Propionibacterium & - & - & - & - & - & $\vee$ & $\vee$ & $\vee$ \\
\hline 10 & 44 & Propionibacterium & - & - & - & - & - & $\vee$ & - & - \\
\hline 11 & 54 & Propionibacterium & - & - & - & - & - & $\vee$ & - & - \\
\hline 12 & 51 & Propionibacterium & - & - & - & - & - & $\vee$ & - & - \\
\hline 13 & 41 & Propionibacterium & - & - & - & - & - & $\vee$ & - & - \\
\hline 14 & 27 & Propionibacterium & - & $\vee$ & $\checkmark$ & - & - & $\vee$ & - & - \\
\hline 15 & 36 & Propionibacterium & Superficial abscess & $\vee$ & - & - & - & $\vee$ & - & - \\
\hline 16 & 26 & Propionibacterium & - & - & - & - & - & - & $\vee$ & $\vee$ \\
\hline 17 & 53 & Proteus & Cellulitis & - & - & - & - & $\vee$ & - & - \\
\hline 18 & 40 & Pseudomonas & Dehiscence & $\vee$ & $\vee$ & $\vee$ & - & $\vee$ & - & $\vee$ \\
\hline 19 & 50 & Pseudomonas & - & v & - & - & $\vee$ & $\vee$ & $\vee$ & $\vee$ \\
\hline 20 & 44 & Serratia marcescens & $\begin{array}{l}\text { Left tissue expander } \\
\text { explant }\end{array}$ & $\vee$ & - & - & - & - & - & $\vee$ \\
\hline 21 & 31 & Serratia marcescens & $\begin{array}{l}\text { Bilateral tissue } \\
\text { expander explant }\end{array}$ & $\vee$ & - & - & - & $\vee$ & $\vee$ & $\vee$ \\
\hline 22 & 57 & $\begin{array}{l}\text { Staphlococcus } \\
\text { epidermidis }\end{array}$ & - & - & - & - & $\vee$ & $\vee$ & - & - \\
\hline 23 & 34 & Staphlococcus warneri & Skin flap necrosis & $\vee$ & - & - & $\vee$ & $\vee$ & $\vee$ & $\vee$ \\
\hline
\end{tabular}


ly, they each had close ties to San Francisco. One patient lived there preoperatively; the other traveled there immediately postoperatively and frequently visited the city before and after surgery. Clinical signs of infection emerged for these two patients between 4 months and 9 months after surgery, despite having previously well healed incisions. Neither patient received radiation therapy, and neither had medical comorbidities such as diabetes, hypertension or smoking.

The first patient, age 31, had had received bilateral immediate tissue expander placement after bilateral modified radical mastectomies. She had a 16 week course of chemotherapy that started one month postoperatively when all incisions were well healed and expansion began. At three months postoperatively, she had transient chemotherapy-induced neutropenia when her white blood cell count (WBC) dropped to 2.5; at that time, 400 $\mathrm{mL}$ of expansion had been completed in each breast. One week after the neutropenic episode, clinical infection in the right breast emerged, and her tissue expander required explantation. Six months after initial tissue expander placement, clinical infection occurred in the left breast, requiring explantation. Cultures at the time of explantation bilaterally grew S. marcescens. Fig. 1, the left breast at the time of explantation, highlights the orangered pigmentation characteristic of $S$. marcescens. The second patient, age 44, had bilateral mastectomies with immediate tissue expander placement and silicone breast implant exchange 6 months later. With chemotherapy, she had both transient neutropenia $(\mathrm{WBC}, 3.5)$ and leukocytosis $(\mathrm{WBC}>36)$ during her expansion period. One month after silicone implant exchange, the left breast developed clinical infection requiring explantation with operative cultures that grew S. marcescens. Both patients

\section{Fig. 1. Tissue expander at the time of explant}

Left breast cavity at the time of tissue expander explant, with orange-red pigmentation of the tissue and expander which is characteristic of Serratia marcescens.

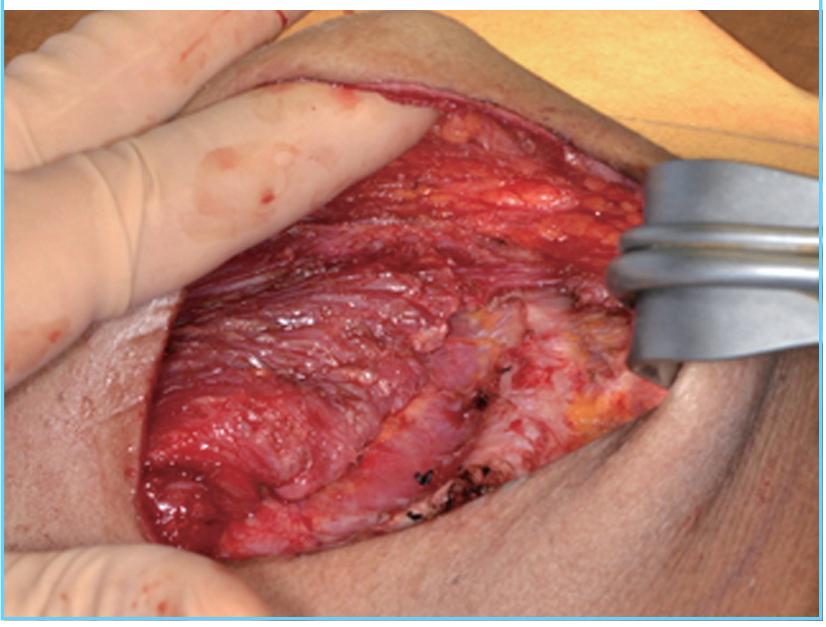

healed well after explantation.

Of the 23 culture positive patients, no others required explantation. Five had clinical cellulitis that responded to antibiotic therapy; these patients were culture positive with Enterococcus, Pseudomonas, MRSA, Staphlococcus, E. Coli and Propionibacterium. Two patients required surgical debridement; both of these patients had ADM tissue expander reconstruction and chemotherapy. All patients all radiation and chemotherapy had been completed after tissue expander placement but before implant exchange.

\section{DISCUSSION}

Our records of implant-based breast reconstruction are uniquely complete because breast capsule cultures were taken for all patients at the time of implant exchange (or explantation, when applicable), regardless of the patient's clinical status. Thus, we were able to highlight common contaminants, as well as bacteria that may become clinically pathologic.

Whether the water in San Francisco is currently colonized by S. marcescens is unknown, as no follow-up studies or reports quantify the burden of $S$. marcescens on public waters and land. Several studies have reported that bodies of water, soil, produce, and insects are natural carriers of $S$. marcescens. Therefore, it is possible that San Franciscans are at higher risk for colonization given the history of military contamination. Though S. marcescens was released into the water over 40 years ago, the bacteria's ability to thrive in moist environments and a wide temperature range may have potentiated its survival to the present day. While S. marcescens optimally grows at 37 degrees Celsius, it can grow in a temperature range from 5 to 40 degrees Celsius [7]. Both patients in this report have had extensive exposure to the water and environment in San Francisco. Therefore, it is possible that they had a higher risk of being colonized by $S$. marcescens relative to the general population.

Biologically, implants that are colonized with S. marcescens have the potential to become a potent nidus for infection. In 1996, Chen et al. [8] published an in vitro and in vivo study of S. marcescens in saline, showing that $S$. marcescens thrives and multiplies exponentially in human saline breast implants for many years. $S$. marcescens survived in vitro in saline-filled implants and proliferated 80 -fold in 7 days, plateauing at the end of 3 weeks. Chemical analyses revealed the presence of glucose in the saline, likely from diffusion across the outer shell of the implant. Chen et al. [8] hypothesized that the survival of $S$. marcescens was partially dependent on the glucose rich environment. As our patients above likely had environmental exposure to $S$. marcescens, the Chen et al. [8] study suggests $S$. marcescens has the potential to proliferate 
in their saline-filled tissue expanders under optimal conditions. If the patients in our report were colonized with $S$. marcescens while healthy, they were likely at higher risk of infection or hematologic seeding from this bacteria while immunocompromised and postsurgery. Furthermore, at a time of infectious vulnerability, both patients had tissue expanders in place that were susceptible to infection [7]. Extensive literature discusses the virulence of $S$. marcescens in opportunistic infections. While the first report of opportunistic $S$. marcescens infection was by Wheat et al. [4] in 1976, over 100 nosocomial outbreaks had been reported by 1977. Common risk factors for S. marcescens infections are invasive instrumentation, extended hospital stay, prolonged antibiotic use, immune compromise, underlying illness, and corticosteroids. The patients in our series had several of the above risk factors, and most importantly were immune compromised at several points of their medical and surgical treatment for breast cancer.

The patients in our report required explantation as a result of their S. marcescens infection. Several recent large scale studies have investigated the relationship between reconstructive implant infection and explantation; however there is no consensus regarding risk factors. McCarthy et al. [9] in 2007 studied 1,170 expander-based reconstructions post mastectomy and found an infection rate of $4.9 \%$. Infection leading to explantation, which occurred in $1.5 \%$ of patients, was associated only with smoking, obesity and hypertension. In 2012, Leyngold et al. [10] published a series of 195 women that showed an infection rate of 5\%, with inpatient procedures as the only significant risk factor. Reported causative bacteria were E. faecalis, MRSA, and Pseudomonas aeruginosa. Patient geography was not reported in these studies.

National US data shows that $S$. marcescens is associated with $1.4 \%$ of bloodstream infections and $3.5 \%$ of nosocomial pneumonia [7]. However, its prevalence in soft tissue infections is not reported locally or nationally. Moreover, given our small sample size and the low incidence of breast infections overall, it is difficult to show statistically significant risk factors for S. marcescens stratified by geography. Nonetheless, close monitoring of patients with geographical risk factors is not burdensome and antibiotic prophylaxis that covers $S$. marcescens , e.g., quinolones, may be considered perioperatively if the surgeon believes that benefits outweigh the harm of a broader spectrum antibiotic. Also, if signs of infection emerge postoperatively for patients with ties to San Francisco, surgeons could have a lower threshold to treat with antibiotics that cover $S$. marcescens.

S. marcescens has a complex history in epidemiology and medicine. Postmastectomy tissue expander-based reconstruction patients are a vulnerable group, as they are immunocompromised to various degrees during their operative course. While the link between the San Francisco $S$. marcescens experiments and opportunistic infection is controversial, several studies have shown that the bacteria has the potential to thrive in the local environment of the city, possibly colonizing the inhabitants. As S. marcescens is a powerful opportunist, these human carriers would likely need to be immunocompromised for colonization to produce adverse effects. A patient's geography is a simple screening tool when considering postoperative risks, especially in the immunocompromised and those receiving prostheses. Closer monitoring for neutropenia during chemotherapy, and a lower threshold to administer $S$. marcescens targeted antibiotics may be warranted in these patients.

\section{REFERENCES}

1. Yu VL. Serratia marcescens: historical perspective and clinical review. N Engl J Med 1979;300:887-93.

2. United States. Congress. Senate. Committee on Human Resources. Subcommittee on Health Scientific Research. Biological Testing Involving Human Subjects by the Department of Defense, 1977: Hearings Before the Subcommittee on Health and Scientific Research of the Committee on Human Resources, United States Senate, Ninety-fifth Congress, First Session ... March 8 and May 23, 1977: US Government Printing Office; 1977.

3. US Department of the Army. US Army Activity in the US Biological Warfare Programs: Vol. II. Annexes. Washington DUDoAPDB.

4. Wheat RP, Zuckerman A, Rantz LA. Infection due to chromobacteria; report of 11 cases. AMA Arch Intern Med 1951; 88:461-6.

5. Fishbach RS, Rosenblatt JE, Dahlgren JG. Pyogenic vertebral osteomyelitis in heroin addicts. Calif Med 1973;119:1-4.

6. Mills J, Drew D. Serratia marcescens endocarditis: a regional illness associated with intravenous drug abuse. Ann Intern Med 1976;84:29-35.

7. Mahlen SD. Serratia infections: from military experiments to current practice. Clin Microbiol Rev 2011;24:755-91.

8. Chen NT, Butler PE, Hooper DC, et al. Bacterial growth in saline implants: in vitro and in vivo studies. Ann Plast Surg 1996;36:337-41.

9. McCarthy CM, Mehrara BJ, Riedel E, et al. Predicting complications following expander/implant breast reconstruction: an outcomes analysis based on preoperative clinical risk. Plast Reconstr Surg 2008;121:1886-92.

10. Leyngold MM, Stutman RL, Khiabani KT, et al. Contributing variables to post mastectomy tissue expander infection. Breast J 2012;18:351-6. 